И. И. Наркевич, Е. В. Фарафонтова, А. А. Рогач, А. А. Кулеш

Белорусский государственный технологический университет

\title{
ПРОВЕРКА СХОДИМОСТИ ИТЕРАЦИОННОЙ ПРОЦЕДУРЫ РЕШЕНИЯ СИСТЕМЫ ИНТЕГРАЛЬНЫХ УРАВНЕНИЙ ДЛЯ ГЕТЕРОГЕННОЙ СИСТЕМЫ «КРИСТАЛЛИЧЕСКАЯ НАНОЧАСТИЦА - ГАЗООБРАЗНАЯ СРЕДА»
}

В работе используется ранее полученная замкнутая система интегральных уравнений и соответствующих алгебраических соотношений, которая описывает структурные и термодинамические характеристики неоднородных (гетерогенных) молекулярных систем. Она получена в рамках двухуровневого статистического метода, который основывается на симбиозе метода коррелятивных функций Боголюбова - Борна - Грина - Кирквуда - Ивона (ББГКИ), метода условных коррелятивных функций Ротта и метода термодинамических функционалов плотности.

Поле плотности в гетерогенной системе с наночастицами задается полем чисел заполнения ячеек, принадлежащих координационным сферам ГЦК решетки, используемой для описания структуры кристаллической наночастицы и окружающей ее газовой среды. Для численного определения профиля поля плотности наночастицы с учетом релаксации параметров ГЦК решетки на ее границе проведена модернизация компьютерной программы, которая ранее была разработана с использованием системы компьютерного проектирования МathCad. Вариационно-статистическим методом с помощью разработанной программы рассчитан профиль поля плотности наночастицы при температуре $\theta=0,6$, которая соответствует равновесию с газообразной средой при температуре ниже тройной точки. Изучена сходимость численного процесса решения полной замкнутой системы интегральных уравнений итерационным методом.

Ключевые слова: двухуровневый статистический метод, вариационный метод, потенциал средних сил, гетерогенная система, наночастица, поле плотности.

Для цитирования: Наркевич И. И., Фарафонтова Е. В., Рогач А. А., Кулеш А. А. Проверка сходимости итерационной процедуры решения системы интегральных уравнений для гетерогенной системы «кристаллическая наночастица - газообразная среда» // Труды БГТУ. Сер. 3, Физикоматематические науки и информатика. 2021. № 2 (248). С. 41-46.

\section{I. Narkevich, E. V. Farafontova, A. A. Rogach, A. A. Kulesh Belarusian State Technological University}

\section{VERIFICATION OF THE CONVERGENCE OF THE ITERATIVE SOLUTION PROCEDURE OF SYSTEM OF INTEGRAL EQUATIONS FOR HETEROGENEOUS SYSTEM «CRYSTALLINE NANOPARTICLE - GASEOUS ENVIRONMENT»}

The paper uses a closed system of statistical equations and formulas that determines the structural and thermodynamic characteristics of ununiform (heterogeneous) molecular systems. It has been obtained using the two-level statistical method, which is a symbiosis of the Bogolyubov - Born - Green - Kirkwood Ivon (BBGKI) method of correlation, the Rott conditional distribution method, and the density thermodynamic functionals method.

The density field in heterogeneous system with nanoparticles is defined with field of filling numbers, belonging to coordination spheres of FCC-lattice being used for characterization the structure of nanoparticle and its surrounding gaseous environment. For numerical derivation of nanoparticle density field profile taking into account the relaxation of FCC-lattice parameters of its boundary, the computer program, developed before on base of MathCad computer-aided design system, has been modified. Using variationstatistical method, with this program was calculated the density field profile at temperature $\theta=0,6$, which is the equilibrium point with gaseous environment below triple point. The convergence of the numerical process of solving a complete closed-loop system of integral equations by the iterative method was investigated.

Key words: two-level statistical method, variation method, potential of average forces, heterogeneous system, nanoparticle, density field.

For citation: Narkevich I. I., Farafontova E. V., Rogach A. A., Kulesh A. A. Verification of the convergence of the iterative solution procedure of system of integral equations for heterogeneous system «crystalline nanoparticle - gaseous environment». Proceedings of BSTU, issue 3, Physics and Mathematics. Informatics, 2021, no. 2 (248), pp. 41-46 (In Russian). 
Введение. Ранее в работах [1-5] была изложена методика решения полной системы интегральных и алгебраических уравнений для гетерогенной системы, содержащей кристаллическую наночастицу внутри флюидной среды (газа или жидкости), которая разработана в рамках двухуровневого статистического метода [6, 7]. Он базируется на совместном использовании метода коррелятивных функций Боголюбова - Борна Грина - Кирквуда - Ивона (ББГКИ), метода условных распределений Ротта [8] и метода термодинамических потенциалов, которые в случае неоднородных систем являются функционалами поля плотности среды.

Двухуровневый статистический метод позволяет реализовать учет неоднородного распределения средних чисел заполнения $n_{i}$ для молекул системы, которые статистически распределены в микроячейках объемами $\omega_{i}$ метода условных распределений Ротта [8], форма и размеры которых претерпевают существенные изменения вблизи границ кристаллических наночастиц. Для этого используется $F_{11}$-приближение, которое учитывает множество наиболее вероятных состояний конденсированной системы из $N$ молекул в объеме $V$, который разделен на микроячейки объемами $\omega_{i}(i=1,2, \ldots, M)$.

Количество микроячеек $M$ в этом приближении превышает число $N$ молекул в наночастице и окружающей ее флюидной среде. Поэтому некоторые микроячейки с определенной вероятностью могут быть вакантными. В результате числа заполнения $n_{i}$ ячеек меньше единицы, а поле их распределения по объему отражает неоднородность поля плотности в объеме наночастицы и окружающей среде.

В двухуровневом статистическом методе используются потенциалы $\varphi_{i j}$ средних сил $[6,7]$, которые в силу неоднородности гетерогенной системы являются функционалами от искомых полей чисел заполнения $n_{p}$ ячеек, центры которых принадлежат координационным сферам с номерами $p(p=1,2, \ldots, P)$ относительно центра сферической наночастицы.

Функционал свободной энергии неоднородной системы зависит от искомого профиля плотности через потенциалы $\varphi_{i j}$ средних сил, которые численно определяются в результате решения достаточно сложной системы статистических интегральных и алгебраических уравнений.

В модифицированной численной методике решения этой системы усреднения потенциала Леннард-Джонса и других функций проводятся по областям локализации функций распределения в виде сфер с радиусами $b_{p}$, внутри которых унарные функции $\hat{F}_{11}^{*}$ считаются постоянными. Эти радиусы связаны со среднеквадратичными отклонениями $\sigma_{p}$ молекул от узлов, принадлежащих координационным сферам с номерами $p$ относительно центра наночастицы.

В результате макроструктура сферической наночастицы с неоднородным радиальным профилем плотности описывается дискретными наборами чисел заполнения $n_{p}$ и радиусов $b_{p}$ cфер. Поэтому искомые потенциалы $\varphi_{i j}$ средних сил оказываются наиболее сильно зависящими от значений чисел $n_{p}$ и радиусов $b_{p}$ сфер в ячейках, которые относятся к ближайшему окружению микроячеек $\omega_{i}$ и $\omega_{j}$ и принадлежат соответствующим координационным сферам с номерами $p$.

Основная часть. Решаемое в работе модифицированное интегральное уравнение для потенциалов средних сил неоднородной однокомпонентной системы с вакансиями имеет вид $[1,2,5]$

$$
\begin{aligned}
& f_{i j}\left(\rho_{i}, b_{j}\right)=n_{j} g_{i j}\left(b_{i}, \rho_{i j}, b_{j}\right) f_{i j}^{(a)}\left(\rho_{i}, b_{j}\right)+ \\
& +\left(1-n_{j} g_{i j}\left(b_{i}, \rho_{i j}, b_{j}\right)\right) f_{i j}^{(a)}\left(b_{i}, \rho_{i j}, b_{j}\right) .
\end{aligned}
$$

Здесь $f_{i j}\left(\rho_{i}, b_{j}\right)=e^{-\beta \varphi_{i j}\left(\rho_{i}, b_{j}\right)}(\beta=1 / \theta-$ обратная температура, $\theta=k T)$, а $\varphi_{i j}\left(\rho_{i}, b_{j}\right)$ - потенциал средней силы, действующий на молекулу в ячейке $\omega_{i}$ со стороны молекулы, равномерно распределенной внутри сферы объемом $v_{j}=(4 / 3) \pi b_{j}^{3}$ в ячейке $\omega_{j} ; \rho_{i}-$ безразмерное расстояние от молекулы в объеме $v_{i}$ до центра объема $v_{j}$, a $g_{i j}$ - аналог радиальной функции для изучаемой гетерогенной системы, представляющей собой совокупность микроячеек, центры которых для молекулярных кристаллов образуют ГЦК решетку:

$$
\begin{gathered}
g_{i j}=\frac{n_{i j}}{n_{i} n_{j}} ; \quad n_{i j}=n_{i}-n_{i j}^{a \mathrm{v}} ; \\
n_{i j}^{a \mathrm{v}}=0,5 B_{i j}+0,5 \sqrt{B_{i j}^{2}+4 n_{i}\left(1-n_{j}\right) A_{i j}^{-1}} ; \\
A_{i j}=f_{i j}^{(a)}\left(b_{i}, \rho_{i j}, b_{j}\right)-1 ; \quad B_{i j}=\left(n_{i}-n_{j}\right)-\frac{1}{A_{i j}},
\end{gathered}
$$

где $\rho_{i j}$ - безразмерное расстояние между центрами объемов $v_{i}$ и $v_{j}$.

Функционалы $f_{i j}^{(a)}\left(\rho_{i}, b_{j}\right)$ и $f_{i j}^{(a)}\left(b_{i}, \rho_{i j}, b_{j}\right)$ определяются следующими выражениями:

$$
\begin{gathered}
f_{i j}^{(a)}\left(\rho_{i}, b_{j}\right)=\frac{1}{v_{j}} \int_{v_{j}} e^{-\beta \Phi\left(\left|\vec{q}_{i}-\vec{q}_{j}\right|\right)} d v_{j} ; \\
f_{i j}^{(a)}\left(b_{i}, \rho_{i j}, b_{j}\right)=\frac{1}{v_{i}} \int_{v_{i}} f_{i j}^{(a)}\left(\rho_{i}, b_{j}\right) d v_{i} .
\end{gathered}
$$

Функционалы энтропии $S$, внутренней энергии $U$ и свободной энергии $F$ сферической наночастицы и окружающей ее флюидной среды рассчитываются по следующим формулам: 


$$
\begin{gathered}
S\left\{n_{p}\right\}=-\sum_{p=1}^{P} Z_{p}\left(n_{p} \ln n_{p}+\left(1-n_{p}\right) \ln \left(1-n_{p}\right)+\right. \\
\left.+\sum_{\substack{j=1 \\
j \neq p}}^{J} n_{p} n_{j} g_{p j} \ln g_{p j}\right) ; \\
U\left\{n_{p}\right\}=\sum_{p=1}^{P} Z_{p} \sum_{\substack{j=1 \\
j \neq p}}^{J}\left(n_{p} n_{j} g_{p j} \psi\left(b_{p}, r_{p j}, b_{j}\right)\right) ; \\
F\left\{n_{p}\right\}=U\left\{n_{p}\right\}-\theta S\left\{n_{p}\right\} .
\end{gathered}
$$

Здесь $Z_{p}$ - число узлов, принадлежащих координационной сфере с номером $p ; J=42$ - число узлов, принадлежащих трем координационным сферам с центрами, совпадающими с центром ячейки $\omega_{p}$, по узлам которых выполняется суммирование в уравнениях (7), (8).

$$
\psi\left(b_{i}, \rho_{i j}, b_{j}\right)=\frac{\int_{v_{i}} \varphi_{i j}^{(a)}\left(\rho_{i}, b_{j}\right) \int_{v_{j}} e^{-\beta \Phi\left(\vec{q}_{i}-\vec{q}_{j}\right)} d v_{j} d v_{i}}{\iint_{v_{i}} e^{-\beta \Phi\left(\left|\vec{q}_{i}-\vec{q}_{j}\right|\right)} d v_{j} d v_{i}} .
$$

Здесь

$$
\varphi_{i j}^{(a)}\left(\rho_{i}, b_{j}\right)=\frac{\int_{v_{j}} \Phi(\rho) e^{-\beta \Phi\left(\left|\vec{q}_{i}-\vec{q}_{j}\right|\right)} d v_{j}}{\int_{v_{j}} e^{-\beta \Phi\left(\left|\vec{q}_{i}-\vec{q}_{j}\right|\right)} d v_{j}} .
$$

В численных расчетах все формулы и уравнения записаны в безразмерном виде, когда геометрические размеры определены в единицах линейного параметра $\sigma$ потенциала Леннард-Джон$\mathrm{ca}$, а все величины, имеющие размерность энергии, определены в единицах энергетического параметра $\varepsilon$ этого же потенциала.

Для сферической наночастицы поле плотности гетерогенной системы зависит только от расстояния $r_{p}$ до центра наночастицы. Поэтому искомый радиальный профиль чисел заполнения $n\left(r_{p}\right)$ аппроксимируется трехпараметрической функцией, содержащей гиперболический тангенс и два вариационных параметра $a$ и $\kappa$, т. е.

$$
n\left(r_{p}\right)=a-\left(a-n_{\infty}\right) \operatorname{th}\left(\kappa \Delta r_{p}\right),
$$

где $n_{\infty}-$ значение чисел заполнения в объемной однородной окружающей среде.

Для сферической поверхности раздела фаз значения вариационных параметров $a$ и $\kappa$ находятся при решении вариационной задачи по отысканию минимума большого термодинамического потенциала $\Omega\left\{n_{p}\right\}=F\left\{n_{p}\right\}-\mu \sum Z_{p} n_{p}$ наночастицы как функционала от искомого радиального профиля чисел заполнения $n_{p}$.

Полная замкнутая система интегральных и алгебраических уравнений (1)-(11) для гетерогенной системы решалась численно методом итераций с помощью модернизированной ранее разработанной компьютерной программы в пакете MathCad. Вариационный расчет параметров $a$ и к для профиля плотности (12) в окрестности сферической кристаллической наночастицы, находящейся в равновесии с газообразной окружающей средой, проводился при температуре $\theta=0,6$, что ниже тройной точки.

Проверка правильности и единственности полученных решений при заданном радиальном профиле плотности была выполнена в результате исследования сходимости разработанной итерационной процедуры.

Сходимость итерационной процедуры к искомому решению системы интегральных уравнений исследована при параметрах $\kappa=6$ и $a=0,045$, которые задают радиальный профиль чисел заполнения $n_{p}$ в газовой среде с кристаллической наночастицей, содержащей 15 координационных сфер. Граница наночастицы соответствует значению номера координационной сферы $p=15$, для которого значение радиуса наночастицы $r_{15}=4,38$ в единицах линейного параметра $\sigma$ потенциала Леннард-Джонса. Например, для аргона $\sigma=3,405 \AA$, следовательно, радиус наночастицы $r_{15}=4,38 \times$ $\times 3,405=14,9 \AA=1,49$ нм.

Для исследования сходимости итерационной процедуры решения замкнутой системы уравнений задавался пробный профиль среднеквадратичных отклонений $\sigma_{p}^{\text {пр }}$, соответствующий макроскопическому кристаллу со значением $\sigma_{p}^{\text {пр }}=$ const, которое отличалось от предполагаемого либо ранее найденного решения для ячейки в центре сферической наночастицы. Проведены две серии итерационных расчетов. В первом случае $\sigma_{p}^{\text {пр }}=0,132$ $\left(b_{p}^{\text {пр }}=0,170\right.$ - параметр, определяющий область локализации унарной функции распределения в ячейке с номером $p$ ), что меньше, чем найденное ранее решение для центральной ячейки $\left(\sigma_{0}=0,149\right.$, $\left.b_{0}=0,192\right)$. Во втором случае $\sigma_{p}^{\text {пр }}=0,183\left(b_{p}^{\text {пр }}=\right.$ $=0,220)$, что больше, чем найденное ранее решение для центральной ячейки $\left(\sigma_{0}=0,149, b_{0}=0,192\right)$. Результаты проведенных итерационных решений системы интегральных уравнений приведены на рис. $1, a, \sigma$.

Из сравнения профилей на рис. $1, a, \sigma$ видно, что как в первом, так и во втором случае профили среднеквадратичных отклонений $\sigma_{p}$ в процессе выполнения итерационной процедуры постепенно деформируются и приближаются к одному и тому же решению, которое практически реализуется уже после 10 -й итерации. Это наглядно видно из анализа соответствующих профилей, изображенных на рис. 2, где итерационные кривые для первого случая пронумерованы числами $i^{*}$, а для второго случая - числами $i$. 

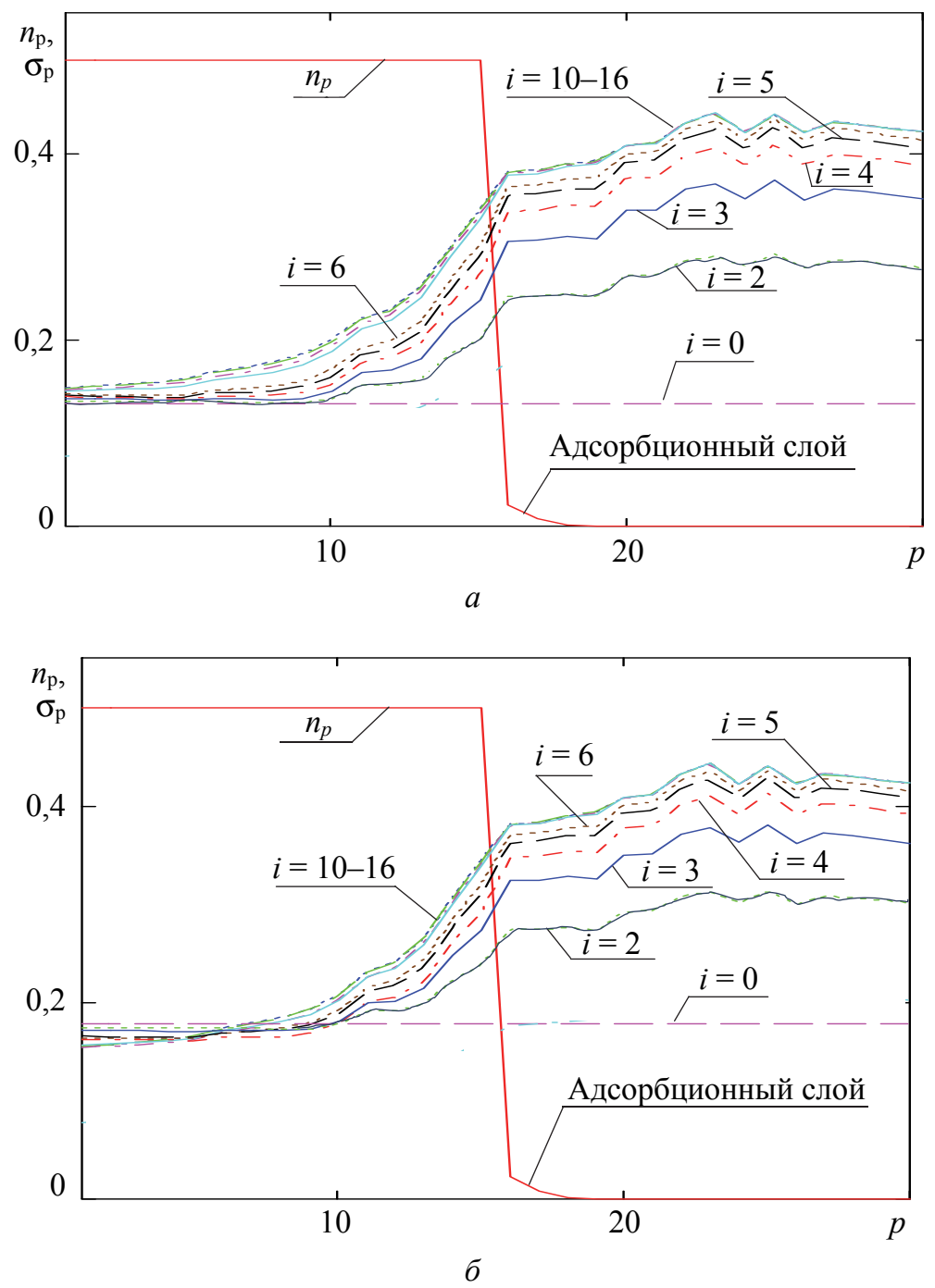

Рис. 1. Заданный радиальный профиль чисел заполнения $n_{p}$ для наночастицы и адсорбционного слоя $\left(\theta=0,6 ; a=0,045 ; \kappa=6 ; n_{\infty}=2 \cdot 10^{-5}\right)$, а также рассчитанные зависимости среднеквадратичных отклонений $\sigma_{p}$ молекул от номеров $p$ координационных сфер для итераций с номерами: $a-i=0,2-6,10-16$ при $\sigma_{p}^{\text {пр }}=0,132 ; \sigma-i=0,2-6,10-16$ при $\sigma_{p}^{\text {пр }}=0,183$

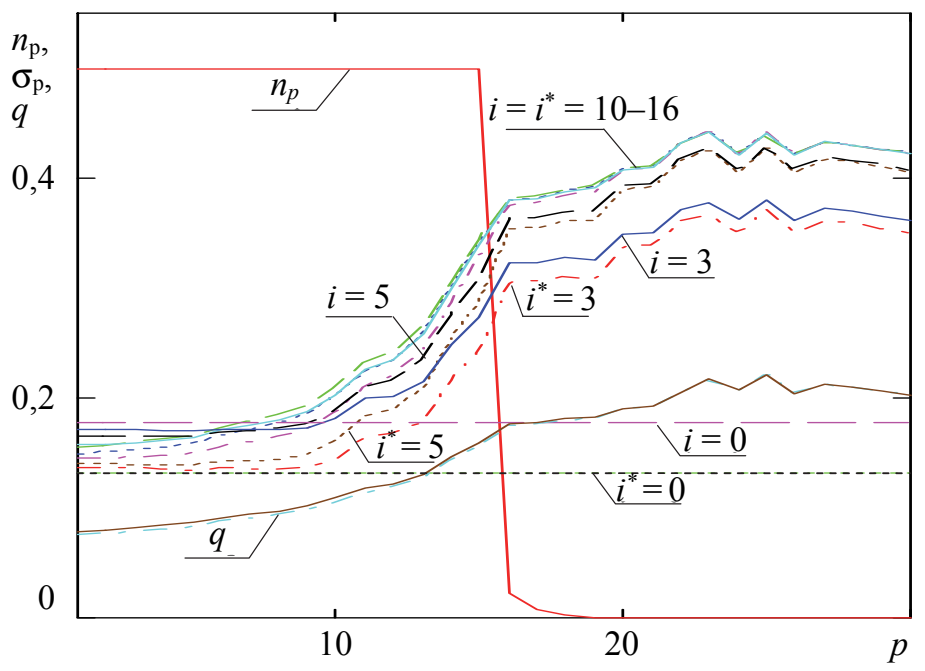

Рис. 2. Заданный радиальный профиль чисел заполнения $n_{p}$ для наночастицы и адсорбционного слоя $\left(\theta=0,6 ; a=0,045 ; \kappa=6 ; n_{\infty}=2 \cdot 10^{-5}\right)$, а также рассчитанные зависимости среднеквадратичных отклонений $\sigma_{p}$ молекул от номеров $p$ координационных сфер для итераций с номерами $i=i^{*}=0,3,5,10-16$ 
Заключение. В работе численно реализована итерационная процедура решения системы интегральных и алгебраических уравнений для заданного равновесного радиального профиля плотности сферической наночастицы с использованием функционалов свободной энергии и большого термодинамического функционала гетерогенной системы: кристаллическая наночастица в равновесии с окружающей объемной газообразной фазой. В результате изучена сходимость численного итерационного процесса решения полной системы уравнений.

\section{Список литературы}

1. Решение модифицированного интегрального уравнения для потенциалов средних сил и расчет параметров фазовых переходов в гетерогенных системах, содержащих кристаллические наночастицы / И. И. Наркевич [и др.] // Труды БГТУ. Сер. 3, Физ.-мат. науки и информатика. 2020. № 2 (236). C. $48-56$.

2. Наркевич И. И., Фарафонтова Е. В. Разработка компьютерной программы для расчета структурных и термодинамических характеристик кристаллических наночастиц разных размеров // Труды БГТУ. Сер. 3, Физ.-мат. науки и информатика. 2019. № 2 (224). С. 34-39.

3. Narkevich I. I., Farafontova E. V. Two-level statistical description of structure of homogeneous macroscopic system and spherical crystalline nanoparticles // Nanoscience and Technology: An International Journal. 2019. № 10 (4). P. 365-376.

4. Статистический расчет термодинамических параметров фазовых переходов и структуры сферических кристаллических наночастиц в гетерогенных молекулярных системах / И. И. Наркевич [и др.] // Фуллерены и наноструктуры в конденсированных средах: сб. науч. ст. Минск, 2020. С. 134-139.

5. Разработка методики установления корреляции между структурой и термодинамическими характеристиками кристаллических наночастиц в рамках двухуровневого молекулярно-статистического метода описания неоднородных систем с учетом релаксации решетки на их границах: науч. исслед. работа / Белорус. гос. технол. ун-т; рук. темы И. И. Наркевич. Минск, 2020. 31 с. № ГР 20192224.

6. Наркевич И. И. Двухуровневый статистический метод описания неоднородных систем. Нордерштедт: LAP LAMBERT Academic Publishing RU, 2019. 114 c.

7. Наркевич И. И. Молекулярно-статистическая теория неоднородных конденсированных сред: дис. ... д-ра физ.-мат. наук. СПб., 1993. 223 с.

8. Ротт Л. А. Статистическая теория молекулярных систем. М.: Наука, 1979. 280 с.

\section{References}

1. Narkevich I. I., Farafontova E. V., Kulesh A. A., Rogach A. A. Solution of the Modified Integral Equation for Medium Force Potentials and Calculation of the Parameters of Phase Transitions in Heterogeneous Systems Containing Crystalline Nanoparticles. Trudy BGTU [Proceedings of BSTU], issue 3, Physics and Mathematics. Informatics, 2020, no. 2 (236), pp. 48-56 (In Russian).

2. Narkevich I. I., Farafontova E. V. Development of a Computer Program for the Calculating of the Structural and Thermodynamic Characteristics of Crystalline Nanoparticles of Different Sizes. Trudy BGTU [Proceedings of BSTU], issue 3, Physics and Mathematics. Informatics, 2019, no. 2 (224), pp. 34-39 (In Russian).

3. Narkevich I. I., Farafontova E. V. Two-level Statistical Description of Structure of Homogeneous Macroscopic System and Spherical Crystalline Nanoparticles. Nanoscience and Technology: An International Journal, 2019, no. 10 (4), pp. 365-376.

4. Narkevich I. I., Farafontova E. V., Kulesh A. A., Rogach A. A. Statistical calculation of Thermodynamic Parameters of Phase Transitions and the Structure of Spherical Crystalline Nanoparticles in Heterogeneous Molecular Systems. Fullereny i nanostruktury v kondensirovannykh sredakh [Fullerenes and nanostructures in condensed matter]. Minsk, 2020, pp. 134-139 (In Russian).

5. Razrabotka metodiki ustanovleniya korrelyatsii mezhdu strukturoy i termodinamicheskimi kharakteristikami kristallicheskikh nanochastits $v$ ramkakh dvukhurovnevogo molekulyarno-statisticheskogo metoda opisaniya neodnorodnykh sistem s uchetom relaksatsii reshetki na ikh granitsakh [Development of a method for establishing a correlation between the structure and thermodynamic characteristics of crystalline nanoparticles within the framework of a two-level molecular-statistical method for describing inhomogeneous systems taking into account the relaxation of the lattice at their boundaries]. Topic leader I. I. Narkevich. Minsk, 2020. 31 p. No. SR 20192224.

6. Narkevich I. I. Dvukhurovnevyy statisticheskiy metod opisaniya neodnorodnykh sistem. Simbioz metodov korrelyativnykh funktsiy i termodinamicheskikh funktsionalov plotnosti [Two-level statistical method for describing heterogeneous systems. Symbiosis of methods of correlative functions and thermodynamic functionals of density]. Norderstedt, LAP LAMBERT Academic Publishing RU, 2019. 114 p. 
7. Narkevich I. I. Molekulyarno-statisticheskaya teoriya neodnorodnykh kondensirovannykh sred. Dis. d-ra fiz.-mat. nauk [Molecular-statistical theory of the non-homogeneous condenced matter. Doct. Diss.]. St. Petersburg, 1993. $223 \mathrm{p}$.

8. Rott L. A. Statisticheskaya teoriya molekulyarnykh sistem [Statistical theory of molecular systems]. Moscow, Nauka Publ., 1979. 280 p.

\section{Информация об авторах}

Наркевич Иван Иванович - доктор физико-математических наук, профессор, профессор кафедры физики. Белорусский государственный технологический университет (220006, г. Минск, ул. Свердлова, 13a, Республика Беларусь). E-mail: narkevich@belstu.by

Фарафонтова Елена Валерьевна - кандидат физико-математических наук, доцент кафедры физики. Белорусский государственный технологический университет (220006, г. Минск, ул. Свердлова, 13а, Республика Беларусь). E-mail: farafontova@belstu.by

Рогач Алеся Александровна - студентка. Белорусский государственный технологический университет (220006, г. Минск, ул. Свердлова, 13а, Республика Беларусь).

Кулеш Алексей Александрович - студент. Белорусский государственный технологический университет (220006, г. Минск, ул. Свердлова, 13а, Республика Беларусь).

\section{Information about the authors}

Narkevich Ivan Ivanovich - DSc (Physics and Mathematics), Professor, Professor, the Department of Physics. Belarusian State Technological University (13a, Sverdlova str., 220006, Minsk, Republic of Belarus). E-mail: narkevich@belstu.by

Farafontova Elena Valer'yevna - PhD (Physics and Mathematics), Assistant Professor, the Department of Physics. Belarusian State Technological University (13a, Sverdlova str., 220006, Minsk, Republic of Belarus). E-mail: farafontova @belstu.by

Rogach Alesya Aleksandrovna - student. Belarusian State Technological University (13a, Sverdlova str., 220006, Minsk, Republic of Belarus).

Kulesh Aleksey Aleksandrovich - student. Belarusian State Technological University (13a, Sverdlova str., 220006, Minsk, Republic of Belarus). 